\title{
TOPOLOGICAL ENTROPY AND DIFFEOMORPHISMS OF SURFACES WITH WANDERING DOMAINS
}

\author{
Ferry Kwakkel and Vladimir Markovic \\ University of Warwick, Mathematics Institute \\ Zeeman Building, Coventry CV4 7AL, United Kingdom; F.H.Kwakkel@warwick.ac.uk \\ University of Warwick, Mathematics Institute \\ Zeeman Building, Coventry CV4 7AL, United Kingdom; V.Markovic@warwick.ac.uk
}

\begin{abstract}
Let $M$ be a closed surface and $f$ a diffeomorphism of $M$. A diffeomorphism is said to permute a dense collection of domains, if the union of the domains are dense and the iterates of any one domain are mutually disjoint. In this note, we show that if $f \in \operatorname{Diff}^{1+\alpha}(M)$, with $\alpha>0$, and permutes a dense collection of domains with bounded geometry, then $f$ has zero topological entropy.
\end{abstract}

\section{Definitions and statement of results}

A result of Norton and Sullivan [8] states that a diffeomorphism $f \in \operatorname{Diff}_{0}^{3}\left(\mathbf{T}^{2}\right)$ having Denjoy-type can not have a wandering disk whose iterates have the same generic shape. By diffeomorphisms of Denjoy-type are meant diffeomorphisms of the two-torus, isotopic to the identity, that are obtained as an extension of an irrational translation of the torus, for which the semi-conjugacy has countably many non-trivial fibers. If these fibers have non-empty interior, then the corresponding diffeomorphism has a wandering disk. Further, by generic shape is meant that the only elements of $\mathrm{SL}(2, \mathbf{Z})$ preserving the shape are elements of $\mathrm{SO}(2, \mathbf{Z})$, such as round disks and squares. In a similar spirit, Bonatti, Gambaudo, Lion and Tresser in [1] show that certain infinitely renormalizable diffeomorphisms of the two-disk that are sufficiently smooth, can not have wandering domains if these domains have a certain boundedness of geometry.

In this note, we study an analogous problem, namely the interplay between the geometry of iterates of domains under a diffeomorphism and its topological entropy. To state the precise result, we first need some definitions. Let $(M, g)$ be a closed surface, that is, a smooth, closed, oriented Riemannian two-manifold, equipped with the canonical metric $g$ induced from the standard conformal metric of the universal cover $\mathbf{P}^{1}, \mathbf{C}$ or $\mathbf{D}^{2}$. We denote by $d(\cdot, \cdot)$ the distance function relative to the metric $g$. Let $\operatorname{Diff}^{r}(M)$ be the group of diffeomorphisms of $M$, where for $r \geq 0$ finite, $f$ is said to be of class $C^{r}$ if $f$ is continuously differentiable up to order $[r]$ and the $[r]$-th derivative is $(r)$-Hölder, with $[r]$ and $(r)$ the integral and fractional part of $r$ respectively. We identity $\operatorname{Diff}^{0}(M)$ with Homeo $(M)$, the group of homeomorphisms of $M$.

Given $f \in \operatorname{Homeo}(M)$, for each $n \geq 1$, define the metric $d_{n}$ on $M$ given by $d_{n}(x, y)=\max _{1 \leq i \leq n}\left\{d\left(f^{i}(x), f^{i}(y)\right)\right\}$. Given $\epsilon>0$, a subset $U \subset M$ is said to be

doi:10.5186/aasfm.2010.3531

2000 Mathematics Subject Classification: Primary 30C62; Secondary 28D20.

Key words: Quasiconformal mappings, entropy, wandering domains.

The first author was supported by Marie Curie grant MRTN-CT-2006-035651 (CODY). 
$(n, \epsilon)$ separated if $d_{n}(x, y) \geq \epsilon$ for every $x, y \in U$ with $x \neq y$. Let $N(n, \epsilon)$ be the maximum cardinality of an $(n, \epsilon)$ separated set. The topological entropy is defined as

$$
h_{\mathrm{top}}(f)=\lim _{\epsilon \rightarrow 0}\left(\lim _{n \rightarrow \infty} \sup \frac{1}{n} \log N(n, \epsilon)\right) .
$$

Next, we make precise the notion of a homeomorphism of a surface permuting a dense collection of domains.

Definition 1.1. Let $S \subset M$ be compact and $\mathscr{D}:=\left\{D_{k}\right\}_{k \in \mathbf{Z}}$ the collection of connected components of the complement of $S$, with the property that $\operatorname{Int}\left(\operatorname{Cl}\left(D_{k}\right)\right)=$ $D_{k}$, where $\mathrm{Cl}(D)$ is the closure of $D$ in $M$. We say $f \in \operatorname{Homeo}(M)$ permutes a dense collection of domains if

(1) $f(S)=S$ and $\mathrm{Cl}\left(D_{k}\right) \cap \mathrm{Cl}\left(D_{k^{\prime}}\right)=\emptyset$ if $k \neq k^{\prime}$,

(2) for every $k \in \mathbf{Z}, f^{n}\left(D_{k}\right) \cap D_{k}=\emptyset$ for all $n \neq 0$, and

(3) $\bigcup_{k \in \mathbf{Z}} D_{k}$ is dense in $M$.

Note that we do not assume a domain to be recurrent, nor do we assume the orbit of a single domain to be dense. A wandering domain is a domain with mutually disjoint iterates under $f$ such that the orbit of the domain is recurrent. Thus a diffeomorphism with a wandering domain with dense orbit is a special case of definition 1.1. Denote $\exp _{p}: T_{p} M \rightarrow M$ the exponential mapping at $p \in M$. The injectivity radius at a point $p \in M$ is defined as the largest radius for which $\exp _{p}$ is a diffeomorphism. The injectivity radius $\iota(M)$ of $M$ is the infimum of the injectivity radii over all points $p \in M$. As $M$ is compact, $\iota(M)$ is positive.

Definition 1.2. (Bounded geometry) A collection of domains $\left\{D_{k}\right\}_{k \in \mathbf{Z}}$ on a surface $M$ is said to have bounded geometry if the following holds: $\mathrm{Cl}\left(D_{k}\right)$ is contractible in $M$ and there exists a constant $\beta \geq 1$ such that for every domain $D_{k}$ in the collection, there exist $p_{k} \in D_{k}$ and $0<r_{k} \leq R_{k}$ such that

$$
B\left(p_{k}, r_{k}\right) \subseteq D_{k} \subseteq B\left(p_{k}, R_{k}\right), \text { with } R_{k} / r_{k} \leq \beta,
$$

where $B(p, r) \subset M$ is the ball centered at $p \in M$ with radius $r>0$. If no such $\beta$ exists, then the collection is said to have unbounded geometry.

By $\mathrm{Cl}\left(D_{k}\right)$ being contractible in $M$ we mean that $\mathrm{Cl}\left(D_{k}\right)$ is contained in an embedded topological disk in $M$. Our definition of bounded geometry is equivalent to the notion of bounded geometry in the theory of Kleinian groups and complex dynamics. It is not difficult, given a surface of any genus, to construct homeomorphisms of that surface with positive entropy that permute a dense collection of domains. We show that producing examples that have a certain amount of smoothness is possible only to a limited degree.

Theorem A. (Topological entropy versus bounded geometry) Let $M$ be a closed surface and $f \in \operatorname{Diff}^{1+\alpha}(M)$, with $\alpha>0$. If $f$ permutes a dense collection of domains with bounded geometry, then $f$ has zero topological entropy.

The outline of the proof of Theorem A is as follows. First we show that the bounded geometry of the permuted domains, combined with their density in the surface, give bounds on the dilatation of $f$ on the complement of the union of the permuted domains. The differentiability assumptions on $f$ allow us to estimate the 
rate of growth of the dilatation on the whole surface $M$. Using a result by Przytycki [9], we show this rate of growth is slow enough so as to ensure the topological entropy of $f$ is zero.

Acknowledgement. We thank the referee for useful comments and suggestions on the manuscript.

\section{Entropy and diffeomorphisms with wandering domains}

First, we study the relation between geometry of domains and the complex dilatation of a diffeomorphism.

2.1. Geometry of domains and complex dilatation. We denote $\lambda$ the measure associated to $g$ and $d \lambda$ the Riemannian volume form. By compactness of $M$, there exists a constant $\kappa>0$ such that

$$
\lambda(B(p, r))=\int_{B(p, r)} d \lambda \geq \kappa r^{2} .
$$

A sequence of positive real numbers $x_{k}$ is called a null-sequence, if for every given $\epsilon>0$ there exist only finitely many elements of the sequence for which $x_{k} \geq \epsilon$. Henceforth, we denote $\ell_{k}:=\operatorname{diam}\left(D_{k}\right)$, the diameter of $D_{k}$ measured in $g$, with $D_{k} \in \mathscr{D}$.

Lemma 2.1. Let $M$ be a closed surface and let $\left\{D_{k}\right\}_{k \in \mathbf{Z}}$ be a collection of mutually disjoint domains with bounded geometry. Then the sequence $\ell_{k}$ is a nullsequence.

Proof. Suppose, to the contrary, that $\left\{D_{k}\right\}_{k \in \mathbf{Z}}$ is not a null-sequence. Then there exist an $\epsilon>0$ and an infinite subsequence $k_{t}$ such that $\operatorname{diam}\left(D_{k_{t}}\right) \geq \epsilon$. By the bounded geometry property, we have that $\operatorname{diam}\left(D_{k_{t}}\right) \leq 2 R_{k_{t}} \leq 2 \beta r_{k_{t}}$ and therefore $r_{k_{t}} \geq \epsilon / 2 \beta$. Therefore, by (2),

$$
\lambda\left(D_{k_{t}}\right) \geq \kappa r_{k_{t}}^{2} \geq \frac{\kappa \epsilon^{2}}{4 \beta^{2}}
$$

for every $t \in \mathbf{Z}$. But this yields that

$$
\sum_{t \in \mathbf{Z}} \lambda\left(D_{k_{t}}\right)=\infty
$$

contradicting the fact that $\lambda(M)<\infty$ as $M$ is compact.

Recall that $S$ is the complement of the union of the permuted domains, i.e. $S=M \backslash \bigcup_{k \in \mathbf{Z}} D_{k}$.

Lemma 2.2. Let $M$ be a closed surface and let $f \in \operatorname{Homeo}(M)$ permute a dense collection $\mathscr{D}$ of domains with bounded geometry. For every $p \in S$, there exists a sequence of domains $D_{k_{t}}$ with $\operatorname{diam}\left(D_{k_{t}}\right) \rightarrow 0$ for $t \rightarrow \infty$ such that $D_{k_{t}} \rightarrow p$.

Proof. Fix $p \in S$ and let $U \subset M$ be an open (connected) neighbourhood of $p$. First assume that $p \in S \backslash \bigcup_{k \in \mathbf{Z}} \partial D_{k}$. This set in non-empty, as otherwise the surface $M$ is a union of countably many mutually disjoint continua; but this contradicts Sierpiński's Theorem, which states that no countable union of disjoint continua is connected. We claim that $U$ intersects infinitely many different elements of $\mathscr{D}$. Indeed, if $U$ intersects only finitely many elements $D_{k_{1}}, \ldots, D_{k_{m}}$, then $\Omega:=\bigcup_{i=1}^{m} \operatorname{Cl}\left(D_{k_{i}}\right)$ is closed. This implies that $U \backslash \Omega$ is open and non-empty, as otherwise $M$ would be 
a finite union of disjoint continua, which is impossible. However, as the union of the elements of $\mathscr{D}$ is dense, $U \backslash \Omega$ can not be open. Thus, there are infinitely many distinct elements $D_{k_{1}}, D_{k_{2}}, \ldots$ of $\mathscr{D}$ that intersect $U$. Taking a sequence of nested open connected neighbourhoods $U_{t}$ containing $p$, we can find elements $D_{k_{t}} \subset U_{t} \backslash U_{t+1}$ for every $t \geq 1$. By Lemma 2.1, $\operatorname{diam}\left(D_{k_{t}}\right)$ is a null-sequence and thus we obtain a sequence of domains $D_{k_{t}}$ with $\operatorname{diam}\left(D_{k_{t}}\right) \rightarrow 0$ for $t \rightarrow \infty$ such that $D_{k_{t}} \rightarrow p$.

As $\operatorname{Int}\left(\mathrm{Cl}\left(D_{k}\right)\right)=D_{k}$, given $p \in \partial D_{k}$ and given any neighbourhood $U \ni p, U$ has non-empty intersection with $M \backslash \mathrm{Cl}\left(D_{k}\right)$. By the same reasoning as above, $p$ is again is a limit point of arbitrarily small domains in the collection $\mathscr{D}$. Thus we have proved the claim for all points $p \in S$ and this concludes the proof.

Next, we turn to the complex dilatation of a diffeomorphism $f \in \operatorname{Diff}(M)$ and its behaviour under compositions of diffeomorphisms, see e.g. [5]. We first consider the case where $f \in \operatorname{Diff}(\mathbf{C})$. The complex dilatation $\mu_{f}$ of $f$ is defined by

$$
\mu_{f}: \mathbf{C} \rightarrow \mathbf{D}^{2}, \quad \mu_{f}(p)=\frac{f_{\bar{z}}}{f_{z}}(p),
$$

and the corresponding differential

$$
\mu_{f}(p) \frac{d \bar{z}}{d z}
$$

is the Beltrami differential of $f$. The dilatation of $f$ is defined by

$$
K_{f}(p)=\frac{1+\left|\mu_{f}(p)\right|}{1-\left|\mu_{f}(p)\right|}
$$

which equals

$$
K_{f}(p)=\frac{\max _{v}\left|D f_{p}(v)\right|}{\min _{v}\left|D f_{p}(v)\right|}
$$

where $v$ ranges over the unit circle in $T_{p} \mathbf{C}$ and the norm $|\cdot|$ is induced by the standard (conformal) Euclidean metric $g$ on $\mathbf{C}$. Denote $[\cdot, \cdot]$ be the hyperbolic distance in $\mathbf{D}^{2}$, i.e., the distance induced by the Poincaré metric on $\mathbf{D}^{2}$. When one composes two diffeomorphisms $f, g: \mathbf{C} \rightarrow \mathbf{C}$, then

$$
\mu_{g \circ f}(p)=\frac{\mu_{f}(p)+\theta_{f}(p) \mu_{g}(f(p))}{1+\overline{\mu_{f}(p)} \theta_{f}(p) \mu_{g}(f(p))},
$$

where $\theta_{f}(p)=\frac{\overline{f_{z}}}{f_{z}}(p)$. It follows that

$$
\mu_{f^{n+1}}(p)=\frac{\mu_{f}(p)+\theta_{f}(p) \mu_{f^{n}}(f(p))}{1+\overline{\mu_{f}(p)} \theta_{f}(p) \mu_{f^{n}}(f(p))} .
$$

We can rewrite (7) as

$$
\mu_{g \circ f}(p)=T_{\mu_{f}(p)}\left(\theta_{f}(p) \mu_{g}(f(p))\right)
$$

where

$$
T_{a}(z)=\frac{a+z}{1+\bar{a} z} \in \operatorname{Möb}\left(\mathbf{D}^{2}\right)
$$

is an isometry relative to the Poincaré metric, for a given $a \in \mathbf{D}^{2}$. Further, the following relation holds

$$
\log \left(K_{g \circ f^{-1}}(f(p))\right)=\left[\mu_{g}(p), \mu_{f}(p)\right] .
$$


To define the complex (and maximal) dilatation of a diffeomorphism of a surface $M$, we first lift $f: M \rightarrow M$ to the universal cover $\widetilde{f}: \widetilde{M} \rightarrow \widetilde{M}$ and denote $\pi: \widetilde{M} \rightarrow M$ be the corresponding canonical projection mapping, where $M=\widetilde{M} / \Gamma$, with $\Gamma$ a Fuchsian group. We assume here that $\widetilde{M}$ is either $\mathbf{C}$ or $\mathbf{D}^{2}$, the trivial case of the sphere $\mathbf{P}^{1}$ is excluded here. As $\pi$ is an analytic local diffeomorphism, $\tilde{f}$ is a diffeomorphism. Further, as $M$ is compact, $f$ is $K$-quasiconformal on $M$ for some $K \geq 1$ and thus $\widetilde{f}$ is $K$-quasiconformal on $\widetilde{M}$. Since $\widetilde{f} \circ h \circ \widetilde{f}^{-1}$ is conformal for every $h \in \Gamma$, it follows from (7) that

$$
\mu_{\tilde{f}}(p)=\mu_{\tilde{f}}(h(p)) \frac{\overline{h_{z}}}{h_{z}}(p) .
$$

In other words, $\mu_{\widetilde{f}}$ defines a Beltrami differential on $\widetilde{M}$ for the group $\Gamma$, or equivalently, it defines a Beltrami differential for $f$ on the surface $M$. Furthermore, the same formulas (5) and (6), defined relative to the canonical (conformal) metric defined on $M$, hold for the dilatation $K_{f}$ of $f$ on $M$.

The following lemma shows that the bounded geometry assumption of the domains has a strong effect on the dilatation of iterates of $f$ on $S$. We say $f$ has uniformly bounded dilatation on $S \subset M$, if $K_{f^{n}}(p)$ is bounded by a constant independent of $n \in \mathbf{Z}$ and $p \in S$.

Lemma 2.3. (Bounded dilatation) Let $M$ be a closed surface and let $f \in$ $\operatorname{Diff}^{1}(M)$ permute a dense collection of domains $\mathscr{D}$. If the collection $\mathscr{D}$ has bounded geometry, then $f$ has uniformly bounded dilatation on $S$.

Proof. Suppose the collection of domains $\mathscr{D}=\left\{D_{k}\right\}_{k \in \mathbf{Z}}$ has $\beta$-bounded geometry for some $\beta \geq 1$. Fix $N \in \mathbf{Z}$ and $p \in S$ and take a small open neigbhourhood $U \subset M$ containing $p$. By Lemma 2.2, there exists a subsequence of domains $D_{k_{t}}$, where $\left|k_{t}\right| \rightarrow \infty$ and $\operatorname{diam}\left(D_{k_{t}}\right) \rightarrow 0$ for $t \rightarrow \infty$ and such that $D_{k_{t}} \rightarrow p$. Denote $q=f^{N}(p) \in S$. We may as well assume that for all $t \geq 1$ the domains $D_{k_{t}}$ are contained in $U$. Define $D_{k_{t}}^{\prime}:=f^{N}\left(D_{k_{t}}\right)$. If we denote $U^{\prime}=f^{N}(U)$, then the sequence $D_{k_{t}}^{\prime}$ converges to $q$ and $D_{k_{t}}^{\prime} \subset U^{\prime}$. By the bounded geometry assumption, for every $t \geq 1$, there exists $p_{t} \in D_{k_{t}}$ and $0<r_{t} \leq R_{t}$ such that

$$
B\left(p_{t}, r_{t}\right) \subseteq D_{k_{t}} \subseteq B\left(p_{t}, R_{t}\right)
$$

with $R_{t} / r_{t} \leq \beta$. As $f \in \operatorname{Diff}^{1}(M)$, the local behaviour of $f^{N}$ around $q$ converges to the behaviour of the linear map $D f_{p}^{N}$. In particular, if we take $p_{t} \in D_{k_{t}}$, then $p_{t} \rightarrow p$ and thus $q_{t}:=f^{N}\left(q_{t}\right) \rightarrow q$, and in order for all $D_{k_{t}}^{\prime}$ to have $\beta$-bounded geometry, it is required that

$$
K_{f^{N}}(p) \leq \frac{R_{t} \beta}{r_{t}}
$$

for $t$ sufficiently large. Indeed, this is easily seen to hold if the map acts locally by a linear map and is thus sufficient as $f \in \operatorname{Diff}^{1}(M)$ and the increasingly smaller domains approach $p$. As $R_{t} / r_{t} \leq \beta$, we must therefore have $K_{f^{N}}(p) \leq \beta^{2}$. As this argument holds for every (fixed) $N \in \mathbf{Z}$ and every $p \in S$, we find $\beta^{2}$ the uniform bound on the dilatation on $S$.

Our smoothness assumptions on $f$ allow us to give bounds on the (complex) dilatation of iterates of $f$ on $M$ in terms of the diameters of the permuted domains. 
Lemma 2.4. (Sum of diameters) Let $M$ be a closed surface and let $f \in$ $\operatorname{Diff}^{1+\alpha}(M)$, with $\alpha>0$, which permutes a collection of domains $\mathscr{D}=\left\{D_{k}\right\}_{k \in \mathbf{Z}}$ with $\beta$-bounded geometry. Then there exists a constant $C=C(\beta)>0$ such that, if $p \in D_{t}$ (for some $t \in \mathbf{Z}$ ) and $q \in \partial D_{t}$, then

$$
\left[\mu_{f^{n+1}}(p), \mu_{f^{n+1}}(q)\right] \leq C \cdot \sum_{s=t}^{t+n} \ell_{s}^{\alpha},
$$

where the domains are labeled such that $f^{s}\left(D_{t}\right)=D_{t+s}$.

To prove Lemma 2.4, we use the following.

Lemma 2.5. Let $f \in \operatorname{Diff}^{1}(M)$ and $p_{0}, q_{0} \in M$. Then

$$
\begin{aligned}
& {\left[\mu_{f^{n+1}}\left(p_{0}\right), \mu_{f^{n+1}}\left(q_{0}\right)\right]} \\
& \leq \sum_{s=0}^{n}\left[T_{\mu_{f}\left(p_{s}\right)}\left(\theta_{f}\left(p_{s}\right) \mu_{f^{n-s}}\left(q_{s+1}\right)\right), T_{\mu_{f}\left(q_{s}\right)}\left(\theta_{f}\left(q_{s}\right) \mu_{f^{n-s}}\left(q_{s+1}\right)\right)\right],
\end{aligned}
$$

where $p_{s}=f^{s}\left(p_{0}\right)$ and $q_{s}=f^{s}\left(q_{0}\right)$.

Proof. Using (9), we write

$$
\left[\mu_{f^{n+1}}\left(p_{0}\right), \mu_{f^{n+1}}\left(q_{0}\right)\right]=\left[T_{\mu_{f}\left(p_{0}\right)}\left(\theta_{f}\left(p_{0}\right) \mu_{f^{n}}\left(p_{1}\right)\right), T_{\mu_{f}\left(q_{0}\right)}\left(\theta_{f}\left(q_{0}\right) \mu_{f^{n}}\left(q_{1}\right)\right)\right] .
$$

By the triangle inequality, we thus have the following inequality

$$
\begin{aligned}
{\left[\mu_{f^{n+1}}\left(p_{0}\right), \mu_{f^{n+1}}\left(q_{0}\right)\right] \leq } & {\left[T_{\mu_{f}\left(p_{0}\right)}\left(\theta_{f}\left(p_{0}\right) \mu_{f^{n}}\left(p_{1}\right)\right), T_{\mu_{f}\left(p_{0}\right)}\left(\theta_{f}\left(p_{0}\right) \mu_{f^{n}}\left(q_{1}\right)\right)\right] } \\
& +\left[T_{\mu_{f}\left(p_{0}\right)}\left(\theta_{f}\left(p_{0}\right) \mu_{f^{n}}\left(q_{1}\right)\right), T_{\mu_{f}\left(q_{0}\right)}\left(\theta_{f}\left(q_{0}\right) \mu_{f^{n}}\left(q_{1}\right)\right)\right] .
\end{aligned}
$$

As both $T_{a}$ (as defined by (10)) and rotations are isometries in the Poincaré disk, we have that

$$
\left[T_{\mu_{f}\left(p_{0}\right)}\left(\theta_{f}\left(p_{0}\right) \mu_{f^{n}}\left(p_{1}\right)\right), T_{\mu_{f}\left(p_{0}\right)}\left(\theta_{f}\left(p_{0}\right) \mu_{f^{n}}\left(q_{1}\right)\right)\right]=\left[\mu_{f^{n}}\left(p_{1}\right), \mu_{f^{n}}\left(q_{1}\right)\right] .
$$

Inequality (14) now follows by induction.

As $\partial D_{t} \subset S$, by Lemma 2.3, $\mu_{f^{n-s}}\left(q_{s+1}\right) \in B_{\delta}$, with $B_{\delta} \subset \mathbf{D}^{2}$ the compact disk centered at $0 \in \mathbf{D}^{2}$ with radius

$$
\delta=\frac{\beta^{2}-1}{\beta^{2}+1} .
$$

Further, define

$$
\delta^{\prime}=\sup _{p \in M}\left|\mu_{f}(p)\right|<1,
$$

and let $B_{\delta^{\prime}} \subset \mathbf{D}^{2}$ be the compact disk centered at $0 \in \mathbf{D}^{2}$ and radius $\delta^{\prime}$.

Lemma 2.6. There exists a constant $C_{1}\left(\delta, \delta^{\prime}\right)$ such that

$$
\left[T_{a}(z), T_{b}(z)\right] \leq C_{1}[a, b],
$$

for given $a, b \in B_{\delta^{\prime}}$ and $z \in B_{\delta}$.

Proof. First we observe that there exists a constant $0<\delta^{\prime \prime}<1$ (depending only on $\delta$ and $\left.\delta^{\prime}\right)$, such that $\left[T_{a}(z), 0\right] \leq \delta^{\prime \prime}$, for every $a \in B_{\delta^{\prime}}$ and every $z \in B_{\delta}$, as the disks $B_{\delta}, B_{\delta^{\prime}} \subset \mathbf{D}^{2}$ are compact. Define $\bar{\delta}=\max \left\{\delta, \delta^{\prime}, \delta^{\prime \prime}\right\}$ and $B_{\bar{\delta}} \subset \mathbf{D}^{2}$ the compact disk with center $0 \in \mathbf{D}^{2}$ and radius $\bar{\delta}$. 
As the Euclidean metric and the hyperbolic metric are equivalent on the compact disk $B_{\bar{\delta}}$, it suffices to show that there exists a constant $C_{1}^{\prime}(\bar{\delta})$ such that

$$
\left|T_{a}(z)-T_{b}(z)\right| \leq C_{1}^{\prime}|a-b|,
$$

where $|z-w|$ denotes the Euclidean distance between two points $z, w \in \mathbf{D}^{2}$. Indeed, if this is shown then (17) follows for a constant $C_{1}$ which differs from $C_{1}^{\prime}$ by a uniform constant depending only on $\bar{\delta}$. To prove (18), we compute that

$$
\left|T_{a}(z)-T_{b}(z)\right|=\left|\frac{(a-b)+(a \bar{b}-\bar{a} b) z+(\bar{b}-\bar{a}) z^{2}}{(1+\bar{a} z)(1+\bar{b} z)}\right| .
$$

As $a, b \in B_{\delta^{\prime}}$ and $z \in B_{\delta}$, there exists a constant $Q_{1}\left(\delta, \delta^{\prime}\right)>0$ so that

$$
|(1+\bar{a} z)(1+\bar{b} z)| \geq Q_{1}^{-1} .
$$

Therefore, it holds that

$$
\left|T_{a}(z)-T_{b}(z)\right| \leq Q_{1}\left(|a-b|+\delta|a \bar{b}-\bar{a} b|+\delta^{2}|a-b|\right) .
$$

In order to prove (18), we show there exists a constant $Q_{2}\left(\delta^{\prime}\right)>0$ such that

$$
|a \bar{b}-\bar{a} b| \leq Q_{2}|a-b| \text {. }
$$

To this end, write $a=r e^{i \phi}$ and $b=r^{\prime} e^{i \phi^{\prime}}$ and $x=a \bar{b}$, so that $x=r r^{\prime} e^{i \nu}$ with $\nu=$ $\phi-\phi^{\prime}$. We may assume that $\nu \in[0, \pi)$. It follows that $a \bar{b}-\bar{a} b=x-\bar{x}=2 i r r^{\prime} \sin (\nu)$. Therefore,

$$
|a \bar{b}-\bar{a} b|=|x-\bar{x}|=2 r r^{\prime}|\sin (\nu)| \leq 2 \delta^{\prime} r|\sin (\nu)|,
$$

as $r^{\prime} \leq \delta^{\prime}$. As the angle between the vectors $a, b \in B_{\delta^{\prime}}$ is $\nu$, it is easily seen that $r|\sin (\nu)| \leq|a-b|$. Combining this estimate with (22), we obtain that

$$
|a \bar{b}-\bar{a} b| \leq 2 \delta^{\prime} r|\sin (\nu)| \leq 2 \delta^{\prime}|a-b| .
$$

Setting $Q_{2}=2 \delta^{\prime}$ yields (21). If we now combine (23) in turn with (20), we obtain a uniform constant

$$
C_{1}^{\prime}\left(\delta, \delta^{\prime}\right)=Q_{1}\left(1+\delta Q_{2}+\delta^{2}\right)
$$

for which (18) holds, as required.

Proof of Lemma 2.4. As $f \in \operatorname{Diff}^{1+\alpha}(M)$, we have that $\mu_{f}(p) \in C^{\alpha}\left(M, \mathbf{D}^{2}\right)$ and $\theta_{f} \in C^{\alpha}(M, \mathbf{C})$, are uniformly Hölder continuous by compactness of $M$. By the triangle inequality, we can estimate the summand in the right-hand side of (14) of Lemma 2.5 as

$$
\begin{aligned}
& {\left[T_{\mu_{f}\left(p_{s}\right)}\left(\theta_{f}\left(p_{s}\right) \mu_{f^{n-s}}\left(q_{s+1}\right)\right), T_{\mu_{f}\left(q_{s}\right)}\left(\theta_{f}\left(q_{s}\right) \mu_{f^{n-s}}\left(q_{s+1}\right)\right)\right]} \\
& \leq\left[T_{\mu_{f}\left(p_{s}\right)}\left(\theta_{f}\left(p_{s}\right) \mu_{f^{n-s}}\left(q_{s+1}\right)\right), T_{\mu_{f}\left(q_{s}\right)}\left(\theta_{f}\left(p_{s}\right) \mu_{f^{n-s}}\left(q_{s+1}\right)\right)\right] \\
& \quad+\left[T_{\mu_{f}\left(q_{s}\right)}\left(\theta_{f}\left(p_{s}\right) \mu_{f^{n-s}}\left(q_{s+1}\right)\right), T_{\mu_{f}\left(q_{s}\right)}\left(\theta_{f}\left(q_{s}\right) \mu_{f^{n-s}}\left(q_{s+1}\right)\right)\right] .
\end{aligned}
$$

To estimate (24), define

$$
z_{s}:=\theta_{f}\left(p_{s}\right) \mu_{f^{n-s}}\left(q_{s+1}\right) \in B_{\delta} \quad \text { and } \quad a_{s}=\mu_{f}\left(p_{s}\right), b_{s}=\mu_{f}\left(q_{s}\right) \in B_{\delta^{\prime}} \subset \mathbf{D}^{2} .
$$

Then (24) reads

$$
\left[T_{\mu_{f}\left(p_{s}\right)}\left(\theta_{f}\left(p_{s}\right) \mu_{f^{n-s}}\left(q_{s+1}\right)\right), T_{\mu_{f}\left(q_{s}\right)}\left(\theta_{f}\left(p_{s}\right) \mu_{f^{n-s}}\left(q_{s+1}\right)\right)\right]=\left[T_{a_{s}}\left(z_{s}\right), T_{b_{s}}\left(z_{s}\right)\right] .
$$

By Lemma 2.6, there exists a constant $C_{1}>0$ such that

$$
\left[T_{a_{s}}\left(z_{s}\right), T_{b_{s}}\left(z_{s}\right)\right] \leq C_{1}\left[a_{s}, b_{s}\right] .
$$


By Hölder continuity of $\mu_{f}$, there exists a constant $\widehat{C}_{1}$ such that

$$
\left[a_{s}, b_{s}\right] \leq \widehat{C}_{1}\left(d\left(p_{s}, q_{s}\right)\right)^{\alpha} .
$$

Therefore, combining equations (27), (28) and (29), we obtain that

$$
\left[T_{\mu_{f}\left(p_{s}\right)}\left(\theta_{f}\left(p_{s}\right) \mu_{f^{n-s}}\left(q_{s+1}\right)\right), T_{\mu_{f}\left(q_{s}\right)}\left(\theta_{f}\left(p_{s}\right) \mu_{f^{n-s}}\left(q_{s+1}\right)\right)\right] \leq \widetilde{C}_{1} \ell_{t+s}^{\alpha},
$$

as $d\left(p_{s}, q_{s}\right) \leq \ell_{t+s}$, with $\widetilde{C}_{1}:=C_{1} \widehat{C}_{1}$.

To estimate (25), we note that the hyperbolic distance and the Euclidean distance are equivalent on the compact disk $B_{\delta}$. Therefore, as the (Euclidean) distance between a point $z \in B_{\delta}$ and $e^{i \phi} z$ is bounded from above by a constant (depending only on $\delta$ ) multiplied by the angle $|\phi|$, by Hölder continuity of $\theta_{f}$ there exists a constant $\widetilde{C}_{2}(\delta)$, such that

$$
\left[\theta_{f}(p) z, \theta_{f}\left(p^{\prime}\right) z\right] \leq \widetilde{C}_{2}\left(d\left(p, p^{\prime}\right)\right)^{\alpha},
$$

for all $z \in B_{\delta}$ and $p, p^{\prime} \in M$, using the local equivalence of the hyperbolic and Euclidean metric. Hence, (25) reduces to

$$
\left.\left[\theta_{f}\left(p_{s}\right) \mu_{f^{n-s}}\left(q_{s+1}\right), \theta_{f}\left(q_{s}\right) \mu_{f^{n-s}}\left(q_{s+1}\right)\right] \leq \widetilde{C}_{2} d\left(p_{s}, q_{s}\right)\right)^{\alpha} \leq \widetilde{C}_{2} \ell_{t+s}^{\alpha},
$$

as $d\left(p_{s}, q_{s}\right) \leq \ell_{t+s}$. Therefore, if we set $C:=\widetilde{C}_{1}+\widetilde{C}_{2}$, then (13) follows.

2.2. Upper bounds on the entropy of a surface diffeomorphism. Next, we relate the topological entropy of a diffeomorphism to its dilatation.

Lemma 2.7. (Entropy and dilatation) Let $M$ be a closed surface and let $f \in$ $\operatorname{Diff}^{1+\alpha}(M)$ with $\alpha>0$. Then

$$
h_{\text {top }}(f) \leq \lim _{n \rightarrow \infty} \sup \frac{1}{2 n} \log \int_{M} K_{f^{n}}(p) d \lambda(p),
$$

with $K_{f}$ the dilatation of $f$.

To prove this we use a result of Przytycki [9]. We need the following notation. Let $L: \mathbf{R}^{m} \rightarrow \mathbf{R}^{m}$ be a linear map and $L^{k \wedge}: \mathbf{R}^{m \wedge k} \rightarrow \mathbf{R}^{m \wedge k}$ the induced map on the $k$-th exterior algebra of $\mathbf{R}^{m} . L^{\wedge}$ denotes the induced map on the full exterior algebra. The norm $\left\|L^{k \wedge}\right\|$ of $L^{k}$ has the following geometrical meaning. Let $\operatorname{Vol}_{k}\left(v_{1}, \ldots, v_{k}\right)$ be the $k$-dimensional volume of a parallelepiped spanned by the vectors $v_{1}, \ldots, v_{k}$, where $v_{i} \in \mathbf{R}^{m}$ with $1 \leq i \leq k$. Then

$$
\begin{aligned}
\left\|L^{k \wedge}\right\| & =\sup _{v_{i} \in \mathbf{R}^{m}} \frac{\operatorname{Vol}_{k}\left(L\left(v_{1}\right), \ldots, L\left(v_{k}\right)\right)}{\operatorname{Vol}_{k}\left(v_{1}, \ldots, v_{k}\right)}, \\
\left\|L^{\wedge}\right\| & =\max _{1 \leq k \leq m}\left\|L^{k \wedge}\right\| .
\end{aligned}
$$

Further, let

$$
\|L\|=\sup _{|v|=1}|L(v)|
$$

the standard norm on operators, with $v \in \mathbf{R}^{m}$ and $|\cdot|$ induced by the corresponding inner product on $\mathbf{R}^{m}$. The following result is due to Przytycki [9] (see also [4]). 
Theorem 2.8. Given a smooth, closed Riemannian manifold $M$ and $f \in \operatorname{Diff}^{1+\alpha}(M)$ with $\alpha>0$. Then

$$
h_{\text {top }}(f) \leq \lim _{n \rightarrow \infty} \sup \frac{1}{n} \log \int_{M}\left\|\left(D f^{n}\right)^{\wedge}\right\| d \lambda(p) .
$$

where $h_{\text {top }}(f)$ is the topological entropy of $f, \lambda$ is a Riemannian measure on $M$ induced by a given Riemannian metric, $\left(D f^{n}\right)^{\wedge}$ is a mapping between exterior algebras of the tangent spaces $T_{p} M$ and $T_{f^{n}(p)} M$, induced by the $D f_{p}^{n}$ and $\|\cdot\|$ is the norm on operators, induced from the Riemannian metric.

Proof of Lemma 2.7. Fix $p \in M$ and let $D f_{p}^{n}: T_{p} M \rightarrow T_{f^{n}(p)} M$. Then

$$
\left\|D f_{p}^{n}\right\|^{2}=K_{f^{n}}(p) J_{f^{n}}(p) .
$$

Thus

$$
\left\|\left(D f_{p}^{n}\right)^{1 \wedge}\right\|=\sqrt{K_{f^{n}}(p) J_{f^{n}}(p)}, \quad \text { and } \quad\left\|\left(D f_{p}^{n}\right)^{2 \wedge}\right\|=J_{f^{n}}(p) .
$$

It follows that

$$
\left\|\left(D f_{p}^{n}\right)^{\wedge}\right\|=\max \left\{\sqrt{K_{f^{n}}(p) J_{f^{n}}(p)}, J_{f^{n}}(p)\right\}
$$

As

$$
\max \left\{\sqrt{K_{f^{n}}(p) J_{f^{n}}(p)}, J_{f^{n}}(p)\right\} \leq \sqrt{K_{f^{n}}(p) J_{f^{n}}(p)}+J_{f^{n}}(p),
$$

we have that

$$
\int_{M}\left\|\left(D f_{p}^{n}\right)^{\wedge}\right\| d \lambda(p) \leq \int_{M}\left(\sqrt{K_{f^{n}} J_{f^{n}}}+J_{f^{n}}\right) d \lambda=\lambda(M)+\int_{M} \sqrt{K_{f^{n}} J_{f^{n}}} d \lambda
$$

as $\lambda(M)=\int_{M} J_{f^{n}} d \lambda$, for every $n \in \mathbf{Z}$. Either $\int_{M} \sqrt{K_{f^{n}} J_{f^{n}}} d \lambda$ is bounded as a sequence in $n$, in which case (32) holds trivially, or the sequence is unbounded in $n$, in which case it is readily verified that

$$
\lim _{n \rightarrow \infty} \sup \frac{1}{n} \log \left(\lambda(M)+\int_{M} \sqrt{K_{f^{n}} J_{f^{n}}} d \lambda\right)=\lim _{n \rightarrow \infty} \sup \frac{1}{n} \log \int_{M} \sqrt{K_{f^{n}} J_{f^{n}}} d \lambda .
$$

By the Cauchy-Schwartz inequality, we have that

$$
\int_{M} \sqrt{K_{f^{n}} J_{f^{n}}} d \lambda \leq \sqrt{\lambda(M)} \cdot \sqrt{\int_{M} K_{f^{n}} d \lambda}
$$

and thus,

$$
\log \int_{M} \sqrt{K_{f^{n}} J_{f^{n}}} d \lambda \leq \frac{1}{2} \log \lambda(M)+\frac{1}{2} \log \int_{M} K_{f^{n}} d \lambda .
$$

It now follows that

$$
\lim _{n \rightarrow \infty} \sup \frac{1}{n} \log \int_{M}\left\|\left(D f^{n}\right)^{\wedge}\right\| d \lambda \leq \lim _{n \rightarrow \infty} \sup \frac{1}{2 n} \log \int_{M} K_{f^{n}} d \lambda
$$

and this proves (32).

2.3. Proof of Theorem A. Let us now complete the proof. Let $f \in \operatorname{Diff}_{A}^{1+\alpha}(M)$, with $\alpha>0$, and suppose that $f$ permutes a dense collection of domains $\left\{D_{k}\right\}_{k \in \mathbf{Z}}$ with bounded geometry. By Lemma 2.1, the sequence $\ell_{k}$ is a null-sequence. Therefore, $\ell_{k}^{\alpha}$ 
is a null-sequence as well, for every $\alpha>0$. Let $p \in D_{t}$ for some $t \in \mathbf{Z}$ and $q \in \partial D_{t}$ and label the domains such that $f^{s}\left(D_{t}\right)=D_{t+s}$. By (11),

$$
\log K_{f^{n}}(f(p))=\left[\mu_{f^{n+1}}(p), \mu_{f}(p)\right]
$$

and thus, by the triangle inequality,

$$
\log K_{f^{n}}(f(p)) \leq\left[\mu_{f^{n+1}}(p), \mu_{f^{n+1}}(q)\right]+\left[\mu_{f^{n+1}}(q), \mu_{f}(p)\right]
$$

As the second term in the right hand side of (39) stays uniformly bounded, we have that

$$
\log K_{f^{n}}(f(p)) \leq\left[\mu_{f^{n+1}}(p), \mu_{f^{n+1}}(q)\right]+C^{\prime}
$$

for some constant $C^{\prime}>0$, independent of $p \in M$ and $n \in \mathbf{Z}$. Define

$$
\xi(n)=\max \sum_{i=0}^{n} \ell_{k_{i}}^{\alpha}
$$

where the maximum is taken over all collections of $n+1$ distinct elements $\left\{D_{k_{0}}, \ldots\right.$, $\left.D_{k_{n}}\right\}$ of $\mathscr{D}$. As $\ell_{k}^{\alpha}$ is a null-sequence, we have that

$$
\lim _{n \rightarrow \infty} \sup \frac{\xi(n)}{n}=0 \text {. }
$$

By Lemma 2.4, we have that

$$
\left[\mu_{f^{n+1}}(p), \mu_{f^{n+1}}(q)\right] \leq C \cdot \sum_{s=t}^{t+n} \ell_{s}^{\alpha},
$$

for some constant $C>0$. Combined with (40), we obtain the following uniform estimate

$$
\log K_{f^{n}}(f(p)) \leq C \xi(n)+C^{\prime},
$$

for every $p \in M$ and $n \in \mathbf{Z}$. Therefore

$$
\begin{aligned}
\log \int_{M} K_{f^{n}} d \lambda & \leq \log \int_{M} \exp \left(C \xi(n)+C^{\prime}\right) d \lambda \\
& =\log \left(\left(\exp \left(C \xi(n)+C^{\prime}\right) \lambda(M)\right)\right. \\
& =C \xi(n)+C^{\prime}+\log (\lambda(M)) .
\end{aligned}
$$

Combining (45) in turn with Lemma 2.7 yields

$$
h_{\text {top }}(f) \leq \lim _{n \rightarrow \infty} \sup \frac{1}{2 n} \log \int_{M} K_{f^{n}} d \lambda \leq C \lim _{n \rightarrow \infty} \sup \frac{\xi(n)}{2 n}=0,
$$

by (41). This proves Theorem A.

\section{Concluding remarks}

Our main result poses the following natural

Question 1. (Differentiable counterexamples) Let $M$ be a closed surface. Do there exist diffeomorphisms $f \in \operatorname{Diff}^{1}(M)$ with positive entropy that permute a dense collection of domains with bounded geometry? 
Remark 1. The referee pointed out that, if $f \in \operatorname{Diff}^{1}(M)$, then $\mu_{f}$ has a modulus of continuity $\eta$; that is

$$
\left[\mu_{f}(p), \mu_{f}(q)\right] \leq \eta(d(p, q)),
$$

where $\eta(\ell) \rightarrow 0$ if $\ell \rightarrow 0$. It follows that, if $f \in \operatorname{Diff}^{1}(M)$, by adapting the proof of Lemma 2.4,

$$
\frac{\left[\mu_{f^{n+1}}(p), \mu_{f^{n+1}}(q)\right]}{n}
$$

is still a null-sequence. However, it is not known whether Przytycki's Theorem holds in the class of $\operatorname{Diff}^{1}(M)$ that would guarantee zero entropy.

Lastly, the following

Remark 2. Oleg Kozlovski and Jean-Marc Gambaudo pointed out that Theorem A can also be derived from Katok's results in [3] about the existence of saddle fixed points for $C^{1+\alpha}$ diffeomorphisms with positive entropy. However, our proof is independent from that in [3]; moreover, it is very likely that our result can be generalized to higher dimensions, whereas the techniques in [3] do not appear to allow for a straightforward generalization to higher dimensions.

\section{References}

[1] Bonatti, C., J. M. Gambaudo, J. M. Lion, and C. Tresser: Wandering domains for infinitely renormalizable diffeomorphisms of the disk. - Proc. Amer. Math. Soc. 122:4, 1994, $1273-1278$.

[2] Fletcher, A., and V. Markovic: Quasiconformal maps and Teichmüller theory. - Oxf. Grad. Texts Math. 11, Oxford Univ. Press, 2007.

[3] Katok, A.: Lyapunov exponents, entropy and periodic orbits for diffeomorphisms. - Publ. Math. Inst. Hautes Études Sci. 51:1, 1980, 137-173.

[4] Kozlovski, O. S.: An integral formula for topological entropy of $C^{\infty}$ maps. - Ergodic Theory Dynam. Systems 18, 1998, 405-424.

[5] Leнto, O.: Univalent functions and Teichmüller spaces. - Grad. Texts in Math. 109, SpringerVerlag, 1987.

[6] McSwiggen, P.: Diffeomorphisms of the torus with wandering domains. - Proc. Amer. Math. Soc. 117:4, 1993, 1175-1186.

[7] Navas, A.: Wandering disks for diffeomorphisms of the $k$-torus: a remark on a theorem by Norton and Sullivan. - Preprint, 2007.

[8] Norton, A., and D. Sullivan: Wandering domains and invariant conformal structures for mappings of he 2-torus. - Ann. Acad. Sci. Fenn. Math. 21, 1996, 51-68.

[9] Przytycki, F.: An upper estimation for topological entropy of diffeomorphisms. - Invent. Math. 59, 1980, 205-213.

Received 14 September 2009 